Doklady Mathematics, 2011, Vol. 84, No. 1, pp. 475-481 (doi: 10.1134/S1064562411040120)

\title{
Algorithms for Searching for Hidden Oscillations in the Aizerman and Kalman Problems
}

\author{
Leonov G.A., Kuznetsov N.V. \\ Draft $^{1} 2$
}

\begin{abstract}
The method of harmonic linearization, describing function method, numerical methods, and the applied bifurcation theory together discover new opportunities for analysis of hidden oscillations of nonlinear control systems. In the present paper new analytical-numerical algorithm for hidden oscillation localization is discussed. Counterexamples construction to Aizerman's conjecture and Kalman's conjecture on absolute stability of control systems are considered.
\end{abstract}

Keywords: Hidden oscillation, describing function method, harmonic balance, absolute stability, Aizerman' conjecture, Aizerman problem, Kalman's conjecture, Kalman problem

\section{Introduction. Aizerman and Kalman conjectures}

In the midpoint of twentieth century M.A. Aizerman [Aizerman (1949)] and R.E. Kalman [Kalman (1957)] formulated two conjectures, which occupy, at once, attention of many famous scholars |Krasovsky (1952); Malkin (1952); Erugin (1952); Pliss (1958); Lefschetz (1965)]. The attempts to refute these conjectures lead to creation of effective methods for the search of hidden oscillations - oscillations which are not "connected" with equilibrium i.e. the creation of numerical procedure of integration of trajectories for the passage from equilibrium to periodic solution is impossible.

Consider a system with scalar nonlinearity

$$
\frac{d \mathbf{x}}{d t}=\mathbf{P} x+\mathbf{q} \varphi\left(\mathbf{r}^{*} \mathbf{x}\right), \quad \mathbf{x} \in \mathbb{R}^{n}
$$

where $\varphi(\sigma)$ is a continuous piecewise-differentiable scalar function and $\varphi(0)=0$. Suppose that for all $k \in$ $\left(\mu_{1}, \mu_{2}\right)$ a zero solution of system (1) with $\varphi(\sigma)=k \sigma$ is asymptotically stable in the large (i.e., a zero solution is Lyapunov stable and any solution of system (1) tends to zero as $t \rightarrow \infty$. In other words, a zero solution is a global attractor of system $(1)$ with $\varphi(\sigma)=k \sigma)$.

In 1949 M.A. Aizerman formulated [Aizerman (1949)] the following conjecture: any system (1) with a nonlinearity, satisfying the property

$$
\mu_{1} \sigma<\varphi(\sigma)<\mu_{2} \sigma \quad \sigma \neq 0
$$

is stable in the large.

The necessary criteria of absolute stability [Leonov et al. (1996)] contradict this hypothesis.

In 1957 R.E. Kalman has formulated a similar hypothesis |Kalman (1957)] with more restrictive condition: if at the points of differentiability of $\varphi(\sigma)$ the condition

$$
\mu_{1}<\varphi^{\prime}(\sigma)<\mu_{2}
$$

is satisfied, then system (1) is stable in the large.

In this work an effective approach for the study of hidden oscillations, based on the union of analytical and numerical methods, is considered.

\footnotetext{
${ }^{1}$ Nikolay V. Kuznetsov, nkuznetsov239 at gmail.com (correspondence author)

${ }^{2} \mathrm{PDF}$ slides http://www.math.spbu.ru/user/nk/PDF/Hidden-oscillation-Absolute-stability-Aizerman-problem-Kalman.pdf
} 


\section{System reduction}

Suppose that matrix $\mathbf{P}$ has a pair of purely imaginary eigenvalues $\pm i \omega_{0}\left(\omega_{0}>0\right)$ and the rest of its eigenvalues have negative real parts (to achieve this one can use harmonic linearization technique).

Then, by nonsingular transformation $\mathbf{x}=$ Sy system (1) can be reduced to the form

$$
\begin{aligned}
& \dot{y}_{1}=-\omega_{0} y_{2}+b_{1} \varphi^{0}\left(y_{1}+\mathbf{c}_{\mathbf{3}}{ }^{*} \mathbf{y}_{3}\right), \\
& \dot{y}_{2}=\omega_{0} y_{1}+b_{2} \varphi^{0}\left(y_{1}+\mathbf{c}_{\mathbf{3}}{ }^{*} \mathbf{y}_{3}\right), \\
& \dot{\mathbf{y}}_{3}=\mathbf{A}_{3} \mathbf{y}_{3}+\mathbf{b}_{3} \varphi^{0}\left(y_{1}+\mathbf{c}_{\mathbf{3}}{ }^{*} \mathbf{y}_{3}\right),
\end{aligned}
$$

where $y_{1}, y_{2}$ are scalar quantities, $\mathbf{y}_{3}$ is $(n-2)$-dimensional vector; $\mathbf{b}_{3}$ and $\mathbf{c}_{\mathbf{3}}$ are $(n-2)$-dimensional vectors, $b_{1}$ and $b_{2}$ are real quantities; $\mathbf{A}_{3}$ is $(n-2) \times(n-2)$-matrix, all eigenvalues of which have negative real parts.

Without loss of generality we assume that for the matrix $\mathbf{A}_{3}$ there exists a positive number $d>0$ such that

$$
\mathbf{y}_{3}^{*}\left(\mathbf{A}_{3}+\mathbf{A}_{3}^{*}\right) \mathbf{y}_{3} \leq-2 d\left|\mathbf{y}_{3}\right|^{2}, \quad \forall \mathbf{y}_{3} \in \mathbb{R}^{n-2}
$$

Write a transfer function of system (1)

$$
\mathbf{r}^{*}(\mathbf{P}-p \mathbf{I})^{-1} \mathbf{q}=\frac{\eta p+\theta}{p^{2}+\omega_{0}^{2}}+\frac{R(p)}{Q(p)}
$$

and a transfer function of system (4)

$$
\frac{-b_{1} p+b_{2} \omega_{0}}{p^{2}+\omega_{0}^{2}}+\mathbf{c}_{\mathbf{3}}^{*}\left(\mathbf{A}_{3}-p \mathbf{I}\right)^{-1} \mathbf{b}_{3}
$$

Here $\mathbf{I}$ is a unit matrix, $\eta$ and $\theta$ are certain real number, $Q(p)$ is a stable polynomial of degree $(n-2), R(p)$ is a polynomial of degree smaller than $(n-2)$. Suppose, the polynomials $R(p)$ and $Q(p)$ have no common roots. By the equivalence of systems (1) and (4), the transfer functions of these systems coincide. This implies the following relations

$$
\begin{array}{r}
\eta=-b_{1}, \quad \theta=b_{2} \omega_{0}, \quad \mathbf{c}_{\mathbf{3}}{ }^{*} \mathbf{b}_{3}+b_{1}=\mathbf{r}^{*} \mathbf{q} \\
\frac{R(p)}{Q(p)}=\mathbf{c}_{\mathbf{3}}{ }^{*}\left(\mathbf{A}_{3}-p \mathbf{I}\right)^{-1} \mathbf{b}_{3} .
\end{array}
$$

\section{Justification of describing function method in critical case}

Let us consider the nonlinearity $\varphi^{0}(\sigma)$ of special form

$$
\varphi^{0}(\sigma)= \begin{cases}\varphi_{1}(\sigma), & \forall|\sigma| \leq \varepsilon \\ \varepsilon^{3} \varphi_{2}(\sigma), & \forall|\sigma|>\varepsilon\end{cases}
$$

The functions $\varphi_{1}(\sigma)$ and $\varphi_{2}(\sigma)$ are the piecewise-differentiable ones, for which the following conditions

$$
\begin{aligned}
& \left|\varphi_{1}(\sigma)\right| \leq \mu|\sigma| \\
& \int_{-\varepsilon}^{\varepsilon}\left(b_{2}\left(\mathbf{c}_{\mathbf{3}}{ }^{*} \mathbf{b}_{3}+b_{1}\right) \varphi_{1}(\sigma)+b_{1} \omega_{0} \sigma\right) \varphi_{1}(\sigma) d \sigma=L \varepsilon^{3}+O\left(\varepsilon^{4}\right)
\end{aligned}
$$

are satisfied. Here $\mu>0$ and $L$ are certain numbers. Further, without loss of generality, we can assume that

$$
\varphi_{2}(\sigma)=0 \quad \forall \sigma \in[-\varepsilon, \varepsilon]
$$


Such conditions are valid, for example, for the following nonlinearity

$$
\varphi^{0}(\sigma)= \begin{cases}\mu \sigma, & \forall|\sigma| \leq \varepsilon \\ M \varepsilon^{3} \operatorname{sign}(\sigma), & \forall|\sigma|>\varepsilon\end{cases}
$$

where $M$ is a certain positive number.

Consider in a phase space of nonlinear system (4) the set

$$
\Omega=\left\{y_{1}+\mathbf{c}_{\mathbf{3}}{ }^{*} \mathbf{y}_{3}=0, \quad y_{2} \in\left[-a_{1},-a_{2}\right], \quad\left|\mathbf{y}_{3}\right| \leq D \varepsilon^{2}\right\}
$$

Here $a_{1,2}$ is a certain positive number, the number $D$ is defined by using the following assertion

Lemma 1 For solutions of system (4) with initial data from $\Omega$ the following representation

$$
\begin{array}{ll}
y_{1}(t)=-\sin \left(\omega_{0} t\right) y_{2}(0)+O\left(\varepsilon^{2}\right), & \\
y_{2}(t)=\cos \left(\omega_{0} t\right) y_{2}(0)+O\left(\varepsilon^{2}\right), & t \in[0, T] \\
\mathbf{y}_{3}(t)=\exp \left(\mathbf{A}_{3} t\right) \mathbf{y}_{3}(0)+\mathbf{O}_{\mathbf{n}-\mathbf{2}}\left(\varepsilon^{2}\right)=\mathbf{O}_{\mathbf{n}-\mathbf{2}}\left(\varepsilon^{2}\right) &
\end{array} \quad t \in
$$

is valid. Besides, there exist numbers $D_{1} \geq D>0$ such that if for small enough $\varepsilon>0$ the inequality

$$
\left|\mathbf{y}_{3}(0)\right| \leq D \varepsilon^{2}
$$

is satisfied, then we have

$$
\left|\mathbf{y}_{3}(T)\right| \leq D \varepsilon^{2}
$$

and

$$
\left|\mathbf{y}_{3}(t)\right| \leq D_{1} \varepsilon^{2}, \quad \forall t \in[0, T]
$$

Consider for trajectories of system (4) Poincare map $F$ of the set $\Omega$ :

$$
F\left\|\begin{array}{l}
y_{1}(0) \\
y_{2}(0) \\
\mathbf{y}_{3}(0)
\end{array}\right\|=\left\|\begin{array}{l}
y_{1}(T) \\
y_{2}(T) \\
\mathbf{y}_{3}(T)
\end{array}\right\|
$$

Here $T$ is a positive number such that

$$
y_{1}(T)+\mathbf{c}_{3}{ }^{*} \mathbf{y}_{3}(T)=0, \quad y_{2}(T)<0
$$

and the relations

$$
y_{1}(t)+\mathbf{c}_{\mathbf{3}}{ }^{*} \mathbf{y}_{3}(t)=0, y_{2}(t)<0, \quad \forall t \in(0, T)
$$

are not valid. Introduce a describing function

$$
\Phi(a)=\int_{0}^{2 \pi / \omega_{0}} \varphi_{2}\left(a \sin \left(\omega_{0} t\right)\right) \sin \left(\omega_{0} t\right) d t .
$$

Theorem 1 If the inequalities

$$
b_{1} \Phi\left(a_{2}\right)>-\frac{2}{\omega_{0}^{2} a_{2}^{2}} L, \quad b_{1} \Phi\left(a_{1}\right)<-\frac{2}{\omega_{0}^{2} a_{1}^{2}} L,
$$

are valid, then for small enough $\varepsilon>0$ the map of Poincare (17) of the set $\Omega$ is mapping in itself: $F \Omega \subset \Omega$. 
In this case by Brouwer's fixed point theorem we have the following statement.

Corollary 1 If inequalities (19) are satisfied, then for small enough $\varepsilon>0$ system (4) has a periodic solution with initial data from $\Omega$. This solution is stable in a sense that its neighborhood $\Omega$ is mapping in itself: $F \Omega \subset \Omega$.

Theorem 2 Suppose that there exists a number $a_{0}>0$ such that the conditions

$$
b_{1} \Phi\left(a_{0}\right)=-\frac{2}{\omega_{0}^{2} a_{0}^{2}} L, \quad a_{0} \neq \nu_{i},\left.\quad b_{1} \frac{d \Phi(a)}{d a}\right|_{a=a_{0}}<\frac{4}{\omega_{0}^{2} a_{0}^{3}} L
$$

are satisfied. Then for small enough $\varepsilon>0$ system (4) has the periodic solution of the form (14) with the initial data

$$
y_{1}(0)=O\left(\varepsilon^{2}\right), y_{2}(0)=-a_{0}+O(\varepsilon), \mathbf{y}_{3}(0)=\mathbf{O}_{\mathbf{n}-\mathbf{2}}\left(\varepsilon^{2}\right)
$$

and with the period

$$
T=\frac{2 \pi}{\omega_{0}}+O\left(\varepsilon^{2}\right)
$$

Corollary 2 The nonlinearity (12)

$$
L=\frac{2}{3}\left(b_{2}\left(\mathbf{c}_{\mathbf{3}}{ }^{*} \mathbf{b}_{3}+b_{1}\right) \mu+b_{1} \omega_{0}\right) \mu, \quad \Phi\left(a_{0}\right)=M \frac{4}{\omega_{0}}
$$

and relation (21) imply that

$$
\begin{aligned}
& y_{1}(0)=O\left(\varepsilon^{2}\right), \\
& y_{2}(0)=-\sqrt{-\frac{\mu}{3 \omega_{0} b_{1} M}\left(b_{2}\left(\mathbf{c}_{\mathbf{3}} * \mathbf{b}_{3}+b_{1}\right) \mu+b_{1} \omega_{0}\right)}+O(\varepsilon), \\
& \mathbf{y}_{3}(0)=\mathbf{O}_{\mathbf{n}-\mathbf{2}}\left(\varepsilon^{2}\right) .
\end{aligned}
$$

A scheme of a proof of theorem 2. The proof of theorem is due to the following Lemmas.

By the form of nonlinearity $\varphi^{0}$ and the representation of solutions (14), for the output of system (4) and the derivative of output we obtain

$$
\begin{aligned}
& \sigma(t)=y_{1}(t)+\mathbf{c}_{\mathbf{3}}{ }^{*} \mathbf{y}_{3}(t)=-\sin \left(\omega_{0} t\right) y_{2}(0)+O\left(\varepsilon^{2}\right), \\
& \dot{\sigma}(t)=\dot{y}_{1}(t)+\mathbf{c}_{3}{ }^{*} \dot{\mathbf{y}}_{3}(t)=-\omega_{0} \cos \left(\omega_{0} t\right) y_{2}(0)+O(\varepsilon) .
\end{aligned}
$$

Hence $|\dot{\sigma}(\tau)|>\kappa>0$ for $|\sigma(\tau)| \leq \varepsilon$. From (23) and (14) it follows that there exist the numbers

$$
0=\tau_{0}<\tau_{1}<\tau_{2}<\tau_{3}<\tau_{4}<\tau_{5}=T
$$

such that (see Fig. 1)

$$
\begin{array}{lll}
\tau_{1}: & \forall t \in\left(0, \tau_{1}\right) \sigma(t) \in(0, \varepsilon), & \sigma\left(\tau_{1}\right)=\varepsilon ; \\
\tau_{2}: & \forall t \in\left(\tau_{1}, \tau_{2}\right) \sigma(t)>\varepsilon, & \sigma\left(\tau_{2}\right)=\varepsilon ; \\
\tau_{3}: & \forall t \in\left(\tau_{2}, \tau_{3}\right) \sigma(t) \in(-\varepsilon, \varepsilon), & \sigma\left(\tau_{3}\right)=-\varepsilon ; \\
\tau_{4}: & \forall t \in\left(\tau_{3}, \tau_{4}\right) \sigma(t)<-\varepsilon, & \sigma\left(\tau_{4}\right)=-\varepsilon ; \\
\tau_{5}=T: & \forall t \in\left(\tau_{4}, T\right) \sigma(t) \in(-\varepsilon, 0), & \sigma(T)=0 .
\end{array}
$$

By the first relation (23) we have the following assertion. 


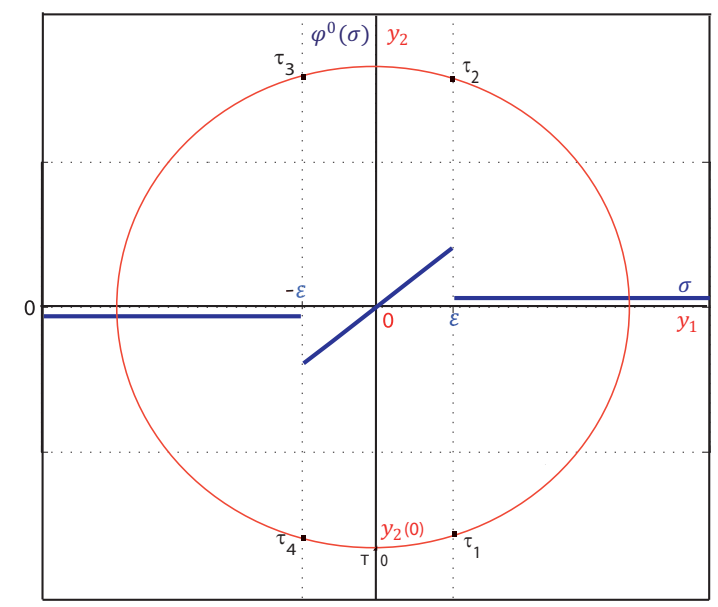

Figure 1: projection of solution on the plane $\left(y_{1}, y_{2}\right)$ and the nonlinearity $\varphi^{0}(\sigma)$ of the form $(12)$

Lemma 2 The following estimations

$$
\begin{aligned}
& \tau_{1}=\frac{\varepsilon}{\omega_{0}\left|y_{2}(0)\right|}+O\left(\varepsilon^{2}\right), \\
& \tau_{2}-\tau_{1}=\frac{\pi}{\omega_{0}}-\frac{2 \varepsilon}{\omega_{0}\left|y_{2}(0)\right|}+O\left(\varepsilon^{2}\right), \\
& \tau_{3}-\tau_{2}=\frac{2 \varepsilon}{\omega_{0}\left|y_{2}(0)\right|}+O\left(\varepsilon^{2}\right), \\
& \tau_{4}-\tau_{3}=\frac{\pi}{\omega_{0}}-\frac{2 \varepsilon}{\omega_{0}\left|y_{2}(0)\right|}+O\left(\varepsilon^{2}\right), \\
& T-\tau_{4}=\frac{\varepsilon}{\omega_{0}\left|y_{2}(0)\right|}+O\left(\varepsilon^{2}\right) .
\end{aligned}
$$

are valid.

Lemma 3 The following estimate

$$
\int_{0}^{2 \pi / \omega_{0}} \varphi_{2}(\sigma(t)) d t=\int_{0}^{2 \pi / \omega_{0}} \varphi_{2}\left(-\sin \left(\omega_{0} t\right) y_{2}(0)\right) d t+O(\varepsilon) .
$$

is valid.

Lemma 4 For small enough $\varepsilon>0$ the estimate

$$
\begin{aligned}
& y_{2}^{2}(T)-y_{2}^{2}(0)= \\
& \quad 2\left|y_{2}(0)\right|\left(\frac{2}{\omega_{0}^{2}\left|y_{2}(0)\right|^{2}} L+b_{1} \Phi\left(\left|y_{2}(0)\right|\right)\right) \varepsilon^{3}+O\left(\varepsilon^{4}\right)
\end{aligned}
$$

is satisfied.

Lemmas 1 and 4 imply that if inequalities (19) are satisfied, then the inclusion $F \Omega \subset \Omega$ occurs. By Brouwer's fixed point theorem, from this inclusion it follows that there exists a fixed point of map $F$ and, consequently, there exists a periodic solution of system (4) with initial data from the set $\Omega$. 


\section{Analytical-numerical method for localization of hidden oscilla- tion}

Based on the above theorems, it is possible to organize a multi-step procedure for the localization of hidden oscillations: initial data obtained in this theorem allow to step aside from stable zero equilibrium and to start numerical localization of possible oscillations [Leonov (2010); Leonov et al. (2010); Bragin et al. (2010)].

Consider for $j=1, \ldots, m$ a finite sequence of piecewise-linear functions

$$
\varphi^{j}(\sigma)=\left\{\begin{array}{ll}
\mu \sigma, & \forall|\sigma| \leq \varepsilon_{j} ; \\
\operatorname{sign}(\sigma) M \varepsilon_{j}^{3}, & \forall|\sigma|>\varepsilon_{j} .
\end{array} \quad \varepsilon_{j}=\frac{j}{m} \sqrt{\frac{\mu}{M}}\right.
$$

Here function $\varphi(\sigma)=\varphi^{m}(\sigma)$ is monotone continuous piecewise-linear function ("saturation").

We choose $m$ in such a way that the graphs of functions $\varphi^{j}$ and $\varphi^{j+1}$ are slightly distinct from each other outside small neighborhoods of points of discontinuity.

Corollary 2 permits one to find stable periodic solution $x(t)=x^{0}(t)$ close to harmonic one of system

$$
\frac{d x}{d t}=P x+q \varphi^{j}\left(r^{*} x\right)
$$

with $j=0$. All the points of this periodic solution may be placed in domain of attraction of stable solution $x^{1}(t)$ of system (30) with $j=1$ or when pass from system (30) with $j=0$ to system (30) with $j=1$, the instability bifurcation destroying periodic solution. In the first case it is possible to find $x^{1}(t)$ numerically, starting a trajectory of system (30) with $j=1$ from the initial point $x^{0}(0)$.

Starting from the point $x^{0}(0)$, after transient process the computational procedure reaches the periodic solution and computes it. In this case the interval $[0, T]$ on which the computation is carried out must be sufficiently large.

After the computation of $x^{1}(t)$ it is possible to take system (30) with $j=2$ and to organize a similar procedure of computing the periodic solution $x^{2}(t)$ by starting from the initial point $x(0)=x^{1}(T)$ a trajectory, which with increasing $t$ may reach the periodic trajectory $x^{2}(t)$ or we observe the instability bifurcation destroying periodic solution.

Proceeding this procedure and computing sequentially the periodic solutions $x^{j}(t)$, using trajectories of system (30) with the initial data $x^{j}(0)=x^{j-1}(T)$, we either obtain a periodic solution of system (30) with $j=m$, either observe, at a certain step, the instability bifurcation destroying periodic solution and stop algorithm.

Similar procedure can be used for localization of hidden attractors (a basin of attraction of which does not contain neighborhoods of equilibria), when the periodic solution sequentially transforms into strange attractor [Leonov et al. (2010); Kuznetsov et al. (2010)].

Suppose that the periodic solution $x^{m}(t)$ of system (30) with monotone and continuous function $\varphi^{m}(\sigma)$ ("saturation") is computed. In this case we organize a similar computational procedure for the sequence of systems

$$
\frac{d x}{d t}=P x+q \psi^{i}\left(r^{*} x\right)
$$

Here $i=0, \ldots, h, \quad \psi^{0}(\sigma)=\varphi^{m}(\sigma)$ and

$$
\psi^{i}(\sigma)=\varphi^{m}(\sigma)+ \begin{cases}0, & \forall|\sigma| \leq \varepsilon_{m} \\ i\left(\sigma-\operatorname{sign}(\sigma) \varepsilon_{m}\right) N, & \forall|\sigma|>\varepsilon_{m}\end{cases}
$$

where $N$ is a certain positive parameter such that $h N<\mu_{2}$. (also here, using the technique of small changes, it is possible to approach various other continuous monotonic increasing functions [Leonov et al. (2010)]).

The finding of periodic solutions $x^{i}(t)$ of system (31) gives a certain counterexample to Kalman's hypothesis for each $i=1, \ldots, h$. 


\section{Counterexamples to Aizerman and Kalman problems}

Consider a system

$$
\begin{aligned}
& \dot{x}_{1}=x_{2}, \\
& \dot{x}_{2}=-x_{4}, \\
& \dot{x}_{3}=x_{1}-2 x_{4}-\varphi\left(x_{4}\right), \\
& \dot{x}_{4}=x_{1}+x_{3}-x_{4}-\varphi\left(x_{4}\right), \\
& \varphi(\sigma)= \begin{cases}5 \sigma, & \forall|\sigma| \leq \frac{1}{5} \\
\operatorname{sign}(\sigma)+\frac{1}{25}\left(\sigma-\operatorname{sign}(\sigma) \frac{1}{5}\right), & \forall|\sigma|>\frac{1}{5}\end{cases}
\end{aligned}
$$

Finding matrix $\mathbf{S}$ and calculating $\mathbf{y}(0)$ by $(22)$, we obtain initial data $\mathbf{x}(0)=\mathbf{S y}(0)=(0,0.2309,0.2309,0)$ for the first step of our algorithm for system (32) with nonlinearity $\varphi^{1}(\sigma)$.

Proceeding the procedure for $j=2, \ldots 10$, we sequentially approximate a periodic solution (see Fig. 2) of initial system $(32)$ with nonlinearity $\varphi(\sigma)$.
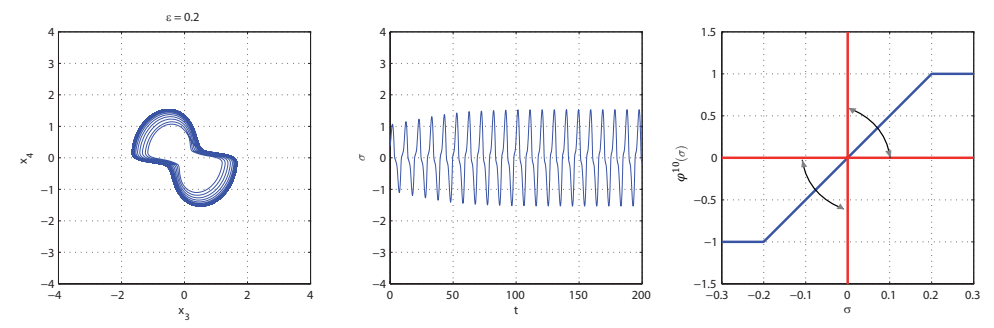

Figure 2: The projection of trajectory on the plane $\left(x_{3}, x_{4}\right)$; system output; nonlinearity and stability sector.

Change the nonlinearity $\varphi(\sigma)$ to the strictly increasing function $\psi^{i}(\sigma)$, where $\mu=1, \varepsilon_{m}=1, N=0.01$. For $\mathrm{i}=3$ after computational process, we obtain periodic solutions (Fig. 3)
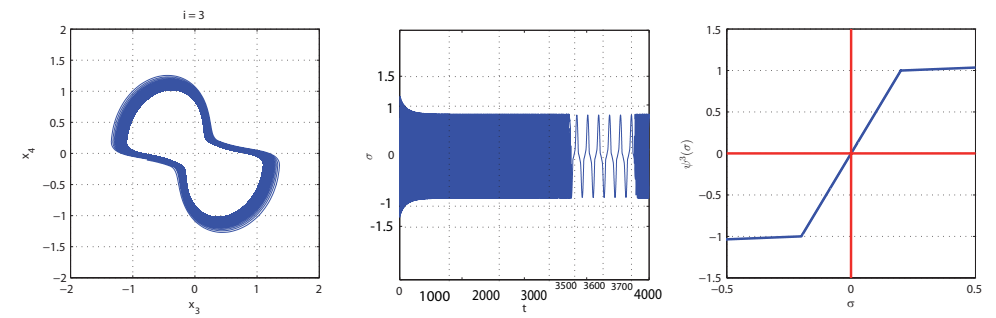

Figure 3: The projection of trajectory on the plane $\left(x_{3}, x_{4}\right)$; system output; nonlinearity and stability sector.

Note that the second part of the algorithm, where saturation zone is rising and counterexample to the problem of Kalman is constructed, complements the numerical results form Barabanov (1988); Bernat \& Llibre (1996) where nonlinearities $\operatorname{sign}(\sigma)$ and $\operatorname{sat}(\sigma)$ are considered respectively.

\section{References}

M.A. Aizerman. On a problem concerning the stability "in the large" of dynamical systems. Russian mathematical surveys, Vol. 4, Iss. 4, 186-188, 1949.

N.E. Barabanov. On the Kalman problem Sib. Math. J., 29(3), 333-341, 1988. 
J. Bernat, J. Llibre. Counterexample to Kalman and Markus-Yamabe conjectures in dimension larger than 3. Dynamics of Continuous, Discrete and Impulsive Systems, Vol. 2, N3. 337-379, 1996.

V.O. Bragin, N.V. Kuznetsov, G.A. Leonov. Algorithm for counterexamples construction for Aizerman's and Kalman's conjectures. IFAC Proceedings Volumes (IFAC-PapersOnline), Volume 4, PART 1, 2010 (doi:10.3182/20100826-3-TR-4016.00008)

N.P. Erugin. A Problem in the theory of stability of automatic control systems. Applied Maht. E Mech., 5 , 620-628, 1952.

R.E. Kalman. Physical and Mathematical mechanisms of instability in nonlinear automatic control systems Transactions of ASME, 79:3, 553-566, 1957.

N.N. Krasovsky. Theorems on the stability of motions determined by a system of two equations. Applied Maht. \& Mech., 16(5), 547-554, 1952.

N.V. Kuznetsov, G.A. Leonov, V.I. Vagaitsev. Analytical-numerical method for attractor localization of generalized Chua's system. IFAC Proceedings Volumes (IFAC-PapersOnline), Volume 4, PART 1, 2010 (doi:10.3182/20100826-3-TR-4016.00009)

S. Lefschetz. Stability of Nonlinear Control Systems.. Academic Press, NY-L, 1965.

G.A. Leonov, I.M. Burkin, A.I. Shepeljavyi. Frequency methods in oscillation theory. Kluwer Acad. Publ., 1996.

G.A. Leonov. On Aizerman problem. Automation and remote control, N7, 37-49, 2009.

G.A. Leonov. Effective methods for periodic oscillations search in dynamical systems. App. math. $\&$ mech., Vol. 74, Iss. 1, 37-73, 2010.

G.A. Leonov, V.O. Bragin, N.V. Kuznetsov.

Algorithm for Constructing Counterexamples to the Kalman Problem. Doklady Mathematics, Vol. 82, No. $1,540-542,2010$.

G.A. Leonov, V.I. Vagaitsev; N.V. Kuznetsov.

Algorithm for localizing Chua attractors based on the harmonic linearization method. Doklady Mathematics, Vol. 82, No. 1, 663-666, 2010.

I.G. Malkin. On the stability of automatic control systems. Applied Maht. \& Mech., 16(4), 495499, 1952.

V.A. Pliss. Some Problems in the Theory of the Stability of Motion. Izd LGU, Leningrad, 1958. 